\title{
Design and Implementation of a Mobile Robot for Carbon Monoxide Monitoring
}

\author{
Wahyu Rahmaniar ${ }^{1}$ and Ardhi Wicaksono ${ }^{1,2}$ \\ ${ }^{1}$ Department of Electrical Engineering, National Central University, Zhongli, Taoyuan, Taiwan \\ ${ }^{2}$ Department of Electronic Engineering and Informatics, Vocational College, Universitas Gadjah Mada, \\ Yogyakarta, Indonesia \\ Email:wahyu.rahmaniar@gmail.com
}

\begin{abstract}
The gas detection problem is relevant to many real-world applications, such as leak detection in industrial settings and landfill monitoring. The mobile robot used for gas detection has several advantages and can reduce danger for humans. In this study, we proposed an integration system for a mobile robot that can be used for carbon monoxide (CO) monitoring with different operating temperatures. The design and implementation of a mobile robot system that proposed consists of the onboard and ground stations. The proposed system can read $\mathrm{CO}$ gas concentration and temperature then send it wirelessly using an XBee module to the ground station. This system was also able to receive the command from the ground station to move the robot. The system provided real-time acquisition data that believed can be a useful tool for monitoring and can be applied for various purposes. The experimental results show that a combination of a mobile robot and environmental sensors can be used for environmental monitoring.
\end{abstract}

Keywords—carbon monoxide, monitoring, robot, sensors, user interface, XBee

\section{INTRODUCTION}

Nowadays, there has been an increase in the use of mobile robots for monitoring. Mobile robots have become an alternative measurement platform for applications that require mobility, real-time data acquisition, and flexible functions [1]-[2]. In the near future, mobile robots can handle most of the public safety, personal privacy, and air pollution. There are several advantages for monitoring using mobile robots, such as object detection [3]-[4], navigation [5], localization [6], and monitoring [7].

The combination of mobile robots and environmental sensors allows automatic supervision of environmental parameters in large areas, such as hazardous materials monitoring [8]. In addition, the mobile robot can provides important information about the environmental situation for early warning alarms [9]. Many kinds of hazardous materials are present in the industrial and general indoor environments, including toxic and harmful gases. Mobile robots equipped with the gas sensors were also used for pollution monitoring and source localization in public areas [10], surveillance of industrial facilities producing harmful gases [11], and monitoring of landfill sites [12].

The mobile robot olfaction (MRO) is a branch of robotics that combines gas sensors with the flexibility of mobile robots. The MRO becomes popular and growing attention, especially in the monitoring of potentially hazardous conditions in recent years [13]. The field of MRO considers two main tasks, such as gas source localization (GSL) and gas distribution mapping (GDM). The GSL purpose is to find the free chemical sources. The GDM purpose is to build a map of the distribution of the chemical in the environment. The GSL algorithms [14]-[15] can be categorized into reactive smoke tracking, smoke modeling, or map-based approaches, which assume that there is one gas source in the environment. The GDM strategy [16] usually requires the robot to explore the entire environment and detect multiple gas sources. Thus, gas-sensitive robots are more advantageous than stationary sensor networks (SN) [17]. SN has a limitation that unable to work well when there is a variation in optimal sensor locations over more considerable distances [18]. In contrast, the mobile robots were able to carry these sensors providing accurate computational and localization resources to develop an online distribution model with adaptive resolution [19].

Previous studies have been using a mobile robot as an early gas leak detection and warning system. Palacin et al. [20] propose the application of an array of MOX gas sensors as the sensor embedded in a personal robot. Martinez et al. [21] presented the measurement of gas concentration and wind intensity using a mobile robot with onboard-sensors that can be used to detected acetone as the target gas source. Rossi et al. [22] presented a modular design of an embedded sensing system and simulated an adaptive sampling algorithm to find gas leaks that minimize the energy required by the sensing element.

This paper focused on carbon monoxide (CO) where the use of a $\mathrm{CO}$ gas sensor in a mobile robot advantages of its lower cost and flexibility [23]. CO gas sensors have limitations in terms of detection range and lack sensitivity to environmental factors such as temperature and humidity [24]. These limitations can be solved by combining $\mathrm{CO}$ gas sensors 
with temperature sensors so that system selectivity can be increased [25]-[26].

$\mathrm{CO}$ is a poisonous gas produced from incomplete carbonbased fuel combustion. If $\mathrm{CO}$ is inhaled, the oxygen flow in the blood will decrease. $\mathrm{CO}$ in high concentrations can cause health problems directly, can even cause death. $\mathrm{CO}$ poisoning can cause long-term health problems in low concentrations if a person experiences regular exposure. Every gas or a propane-based engine will produce $\mathrm{CO}$. Homeowners can suffer from $\mathrm{CO}$ poisoning if they are in danger from sources such as gas-fired stoves and water heaters, blocked fireplaces and chimneys, cars running in attached garages, and burning fuels indoors [27].

Based on the studies above, this paper proposes an integrated system consisting of hardware, mechanics, and user interfaces to detect $\mathrm{CO}$ gas leaks. The hardware contains a mainboard that used to integrate several components. The mechanics are four wheels mobile robots. The user interface provided to receive data from the mobile robot and send a command to the mobile robot.

The remainder of the paper is organized as follows. Section II introduces the proposed method. Section III, IV, and $\mathrm{V}$ describe the robot hardware, sensors used for monitoring, and the ground station, respectively. Section VI presents the experimental results. Finally, conclusions are drawn in Section VII.

\section{THE PROPOSED SYSTEM}

This section introduces the proposed system design. Fig. 1 shows an overview of the proposed monitoring consists of onboard systems and a ground station. The XBee module installed in the ground station system to connect with the PC via RS-232 serial. on-board system consist of carbon monoxide (CO) gas sensor (Fig. 2(a)), temperature sensor (Fig. 2(b)), an XBee module (Fig. 2(c)), LCD 16×2 (Fig. 2(d)), and a DC motor (Fig. 2(e).

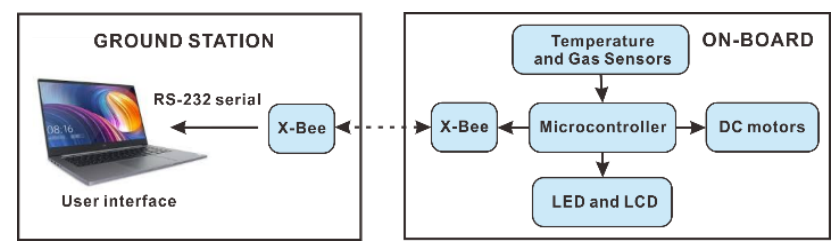

Fig. 1. System overview.

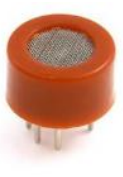

(a)

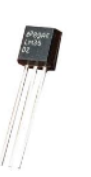

(b)

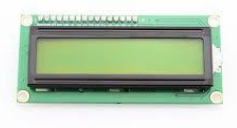

(d)

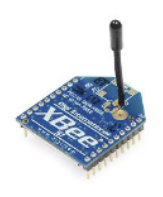

(c)

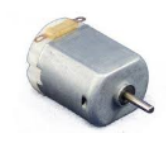

(e)

Fig. 2. On-board components. (a) CO gas. (b) Temperature sensor. (c) XBee module. (d) LCD. (e) DC motor.

\section{ROBOT DESIGN}

Table I summarizes pin connections on $\mathrm{CO}$ gas sensor [28], temperature sensor [29], LCD, an XBee module [30], and motor DC to the ATmega32 AVR microcontroller. Table II defines the sensors and communication device specifications.

TABLE I. PINS CONNECTION OF THE COMPONENTS

\begin{tabular}{|l|l|l|}
\hline Component Name & Component Pin & \multicolumn{1}{|c|}{ ATmega32 Port } \\
\hline LCD & Display pin & Port C \\
\hline LED & Input & Port D \\
\hline MQ9 & Output analog & ADC Input 0 \\
\hline LM35 & Output analog & ADC Input 1 \\
\hline L298 & Motor control & Port B \\
\hline XBee & RX-TX & UART \\
\hline Relay & Input & Port C \\
\hline
\end{tabular}

TABLE II. SENSORS SPECIFICATION

\begin{tabular}{|l|l|l|l|}
\hline \multicolumn{1}{|c|}{$\begin{array}{c}\text { Component } \\
\text { Name }\end{array}$} & $\begin{array}{c}\text { Supply } \\
\text { (VDC) }\end{array}$ & \multicolumn{1}{|c|}{$\begin{array}{c}\text { Power } \\
\text { (mA) }\end{array}$} & \multicolumn{1}{c|}{ Range } \\
\hline MQ9 & 1.5 and 5 & 100 & 10 to 1000ppm \\
\hline LM35 & 5 & 500 & -55 to 150 Celsius \\
\hline XBee & 9 & 250 & $100 \mathrm{~m}$ \\
\hline
\end{tabular}

The schematic diagram of the connection of the components is shown in Fig. 3. ATmega32 [31] is a lowpower microchip 8-bit AVR RISC-based microcontroller that combines 32KB ISP flash memory, 2KB SRAM, and 1024Bytes EEPROM. ATmega32 has 32 general-purpose I/O lines, 4 pins pulse-width modulations (PWM), 8 channel 10bit ADC, a serial programmable USART, 32 general-purpose registers, real-time counters, 3 flexible timer/counters, 2 -wire serial interfaces, and the JTAG interface for on-chip debugging.

The three LEDs, as shown in Fig. 3 were used as an indicator for $\mathrm{CO}$ concentration, i.e., green, yellow, and red, which represents normal, medium, and high, respectively. LCD is used to display the $\mathrm{CO}$ concentration in parts per million (ppm) unit and temperature in the Celsius unit. Two relays are used to control voltage input for the $\mathrm{CO}$ gas sensor

In the system, ATmega32 sends pulse width modulation (PWM) information to control the speed of DC motor through L298 [32]. L298 is a dual full-bridge to control bi-directional DC motor. The rotation direction controlled using an $\mathrm{H}-$ Bridge that causes the current flow direction through the DC motor is inverted, as shown in Fig. 4.

Fig. 5 shows the XBee connection and the power supply circuit. The voltage for the mobile robot is $9 \mathrm{~V}$ DC that is converted into $5 \mathrm{~V}$ DC by a voltage converter and the voltage of the XBee module is converted to 3.3V DC. The LED is used as an indicator for data transmission using XBee. 


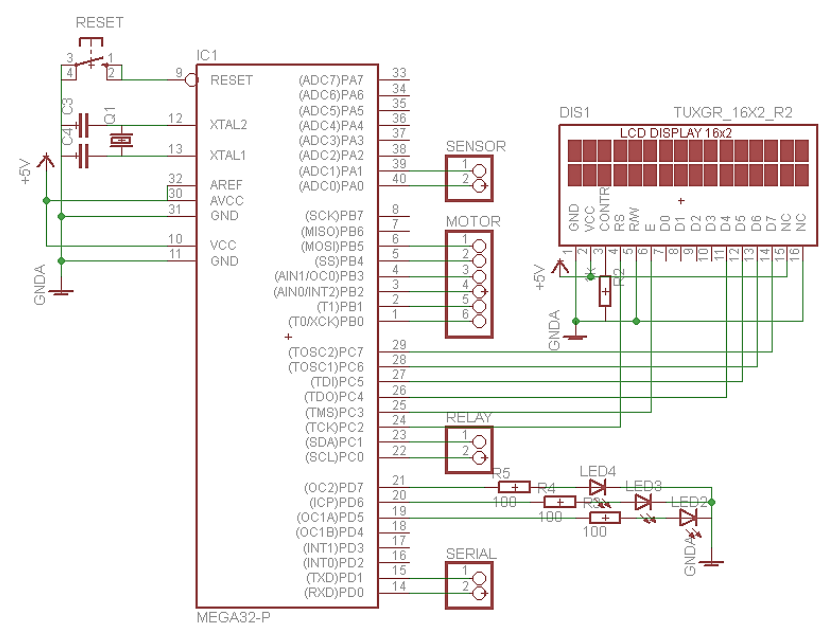

Fig. 3. Components connection to the microcontroller.

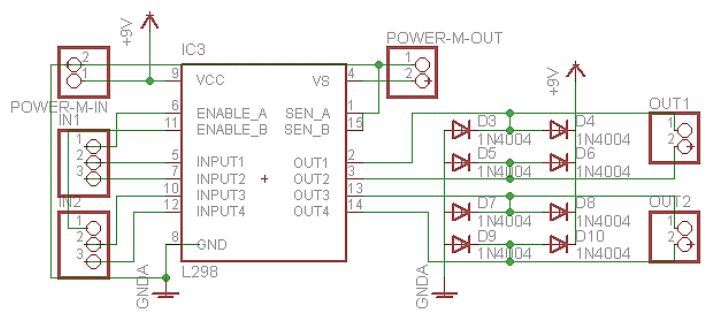

Fig. 4. L298 module connection.

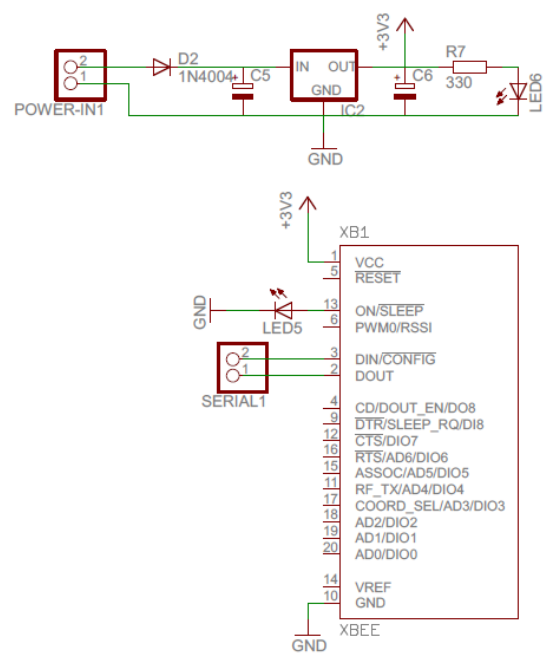

Fig. 5. XBee module connection.

An XBee module provides a UART interface to transmit (TX) and receive (RX) data that are connected to the UART pins on the ATmega32. The XBee module operates using ZigBee protocol with a low-power wireless sensor network, which requires minimal power and provides reliable data transmission between remote devices.

\section{SENSORS FOR MONITORING}

\section{A. CO gas sensor}

The MQ9 gas sensor [27] operates by method of cycle high and low temperature and detect $\mathrm{CO}$ when the low temperature. Fig. 6 shows the basic circuit of MQ9. This sensor requires two kinds of voltage: heater voltage $(\mathrm{VH})$ and reference voltage $(\mathrm{Vc})$. The $\mathrm{VH}$ used to supply certified working temperature to the sensor, while Vc used to detect voltage (VRL) on load resistance (RL) that assembled series with the sensor. Fig. 7 shows the DC/DC voltage converter to convert $5 \mathrm{~V}$ DC into $1.5 \mathrm{~V}$ DC as $\mathrm{VH}$ for MQ9

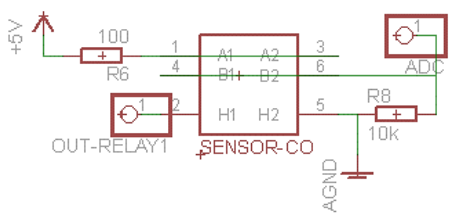

Fig. 6. MQ9 basic circuit.

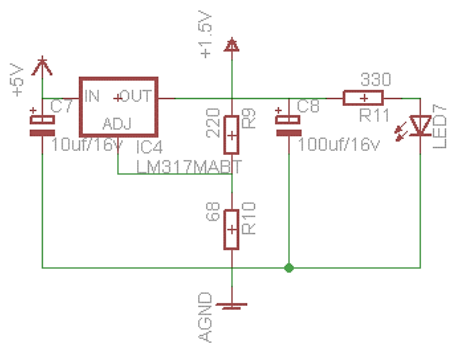

Fig. 7. DC/DC voltage converter.

The MQ9 requires 48 hours of preheat time to able for testing. The resistance ratio of the sensor can be calculated as $R s / R o$. Ro means the resistance of the sensor in the environment of $1000 \mathrm{ppm}$ Propane. Rs means the resistance of the sensor in 1000ppm Propane under different temperatures and humidity. Rs can be calculated as follows

$$
R s=\left(\frac{V c}{V_{R L}}-1\right) \times R L
$$

where $V c$ is a reference voltage $5 \mathrm{~V} \mathrm{DC}, V_{R L}$ is the voltage output of the MQ9, and $R L$ is 10 (10 K Ohm resistor). Based on the MQ9 datasheet [27], ppm value of the CO concentration can be calculated as follows

$$
p p m=\sqrt[-0.53]{\frac{R s}{30.34 \times R o}}
$$

The relay circuit, as shown in Fig. 8, used to drive voltage for the MQ9 sensor. This circuit work by switching input voltage for the MQ9 sensor from $5 \mathrm{~V}$ DC to $1.5 \mathrm{~V}$ DC and vice versa. The $V R L$ will be read after MQ9 sensor is getting $5 \mathrm{~V}$ DC for 60 seconds and $1.5 \mathrm{~V}$ DC for 90 seconds.

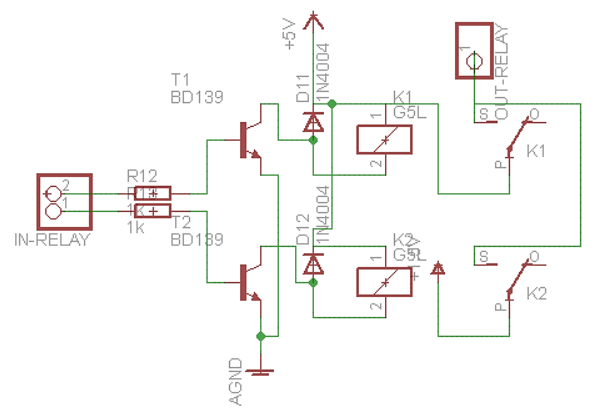

Fig. 8. Relay module connection. 


\section{B. Temperature sensor}

Temperature measurement, which affects most physical and electronic systems, is often a parameter measured in industrial processes [33]. The LM35 temperature sensor [28] does not require any external calibration to provide typical accuracies of 0.25 Celsius degree at room temperature. Fig. 9 shows the basic circuit of LM35.

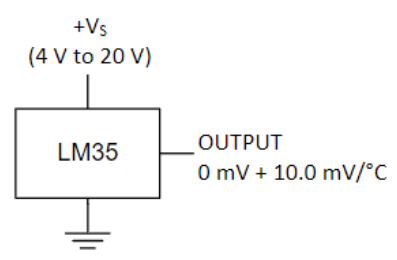

Fig. 9. LM35 basic circuit [28]. by

The temperature in the Celsius degree can be calculated

$$
\text { temperature }=\frac{V_{O U T}}{10}
$$

where $V_{\text {OUT }}$ is the LM35 voltage output in $\mathrm{mV}$.

\section{THE GROUND STATION}

The circuit design of the XBee module on the ground station shown in Fig 10. The LED indicator is connected to an RSSI pin that will flash when the data is sent. The DIN pin has a function to receive data from the TX pin on the robot. The DOUT pin has a role in sending data to the RX pin on the robot. A reset pin is pulled up by a $10 \mathrm{k}$ Ohm resistor. The $\mathrm{XBee}$ module on the onboard system is defined master node, whereas the XBee module on the ground station is defined as a slave node. The slave mode only receives data from the master IP address. The XBee module communicates with the PC via RS232 using the Max232 chip and DB9 connectors.

The proposed user interfaces, as shown in Fig. 11 developed using Visual Basic.Net. The user interface has a features store and displays $\mathrm{CO}$ concentration and temperature in real-time. The user interfaces able to send commands to the DC motors to move the robot.

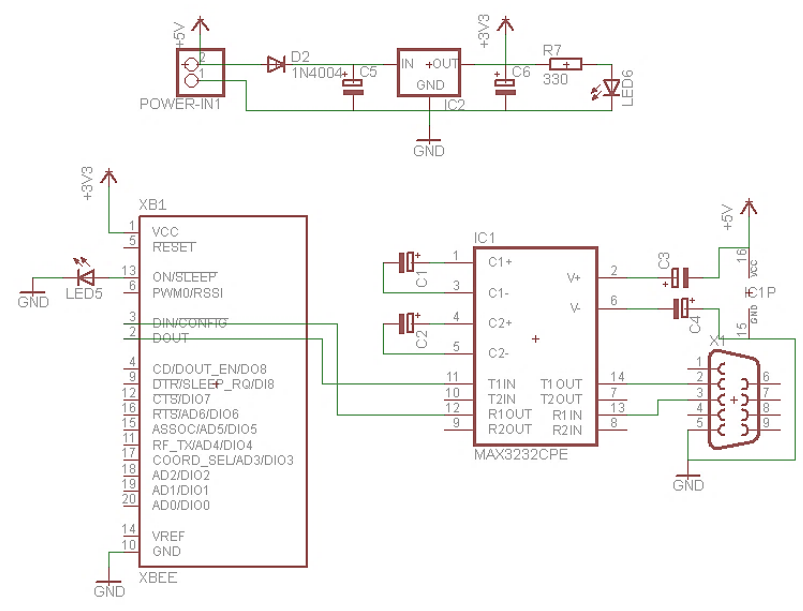

Fig. 10. XBee module connection in the ground station.

In the user interface, three indicators to represent the $\mathrm{CO}$ level warnings are used, i.e., green, yellow, and red as normal, medium, and high, respectively. If the CO ppm is less than 25 , the green indicator will turn on which means the air is normal and clean. If the CO ppm is between 25 to 50 , the yellow indicator will turn on which means the air is starting to be exposed to a little $\mathrm{CO}$. If the $\mathrm{CO}$ ppm is more than 50, the red indicator will turn on which means that the air is exposed to high levels of $\mathrm{CO}$ and can be harmful to health.

The user interface can send commands to move the mobile robot forward, backward, turn left, turn right, and stop, sent as characters $A, B, C, D$, and $E$, respectively. These commands are changed in the mobile robot to provide output to IC L298 for Enable (EN) A and B pins, and Input (IN) 1 to 4 pins. Table III explains robot control commands sent from the ground station to move the robot. Fig. 12 shows an algorithm for communication between the mobile robot and the ground station using the XBee module.

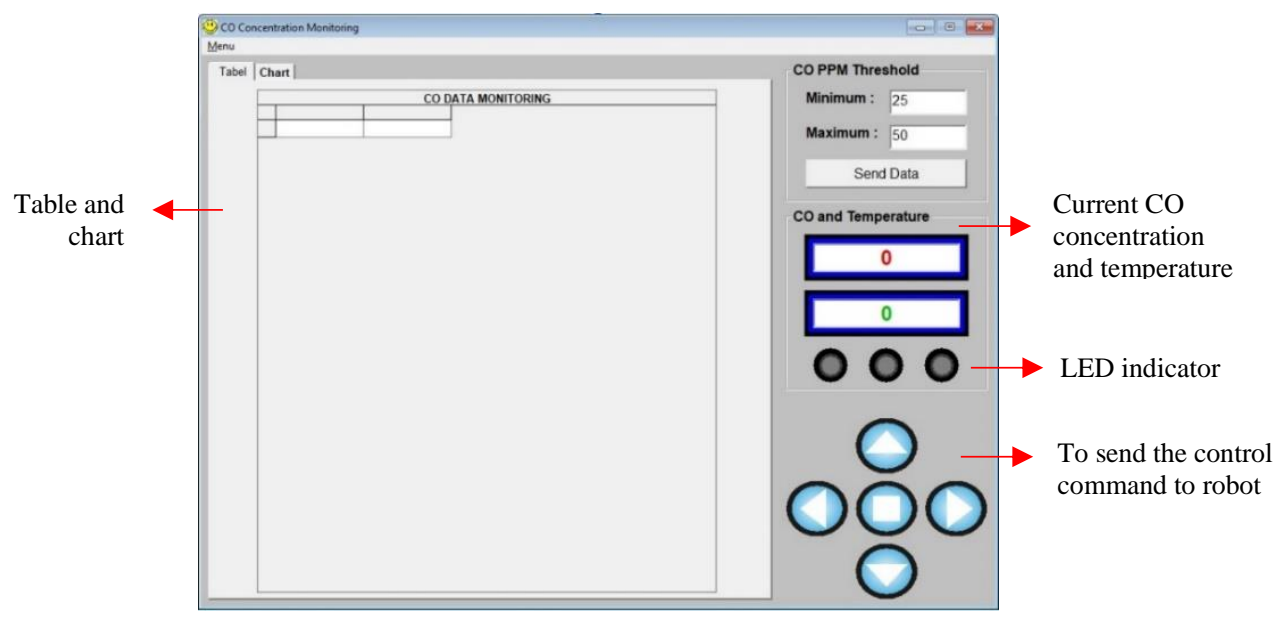

Fig. 11. The proposed user interface. 
TABLE III. ROBOT CONTROL COMMAND

\begin{tabular}{|l|c|c|c|c|c|c|c|}
\hline \multirow{2}{*}{ Command } & \multirow{2}{*}{ Char } & \multicolumn{6}{|c|}{ Output to IC L298 } \\
\cline { 3 - 8 } & & ENA & ENB & IN1 & IN2 & IN3 & IN4 \\
\hline Forward & A & 1 & 1 & 0 & 1 & 1 & 0 \\
\hline Backward & B & 1 & 0 & 1 & 1 & 0 & 1 \\
\hline Turn left & C & 1 & 1 & 0 & 1 & 0 & 1 \\
\hline Turn right & D & 1 & 0 & 1 & 1 & 1 & 0 \\
\hline Stop & E & 0 & 0 & 0 & 0 & 0 & 0 \\
\hline
\end{tabular}

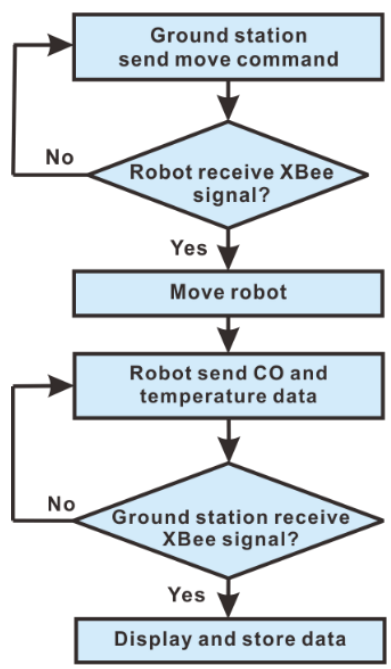

Fig. 12. Algorithm for communication between robot and ground station.

\section{RESULTS AND DISCUSSION}

Fig. 13 shows the implementation of the mobile robot consists of the $\mathrm{CO}$ gas and temperature sensors, an XBee module, LCD, and driver for DC motors. The lithium battery used as a power supply can last for an hour, depending on the speed of the robot.

The ground station as a monitoring interface is shown in Fig. 14. The XBee module can transmit data outdoors with a range of around $1000 \mathrm{~m}$ in outdoor situation and $90-100 \mathrm{~m}$ in indoor situations, with the data transmission speed (baud rate) used is 9600 bits per second (bps). Fig. 15 shows the results of $\mathrm{CO}$ gas and temperature data monitoring.

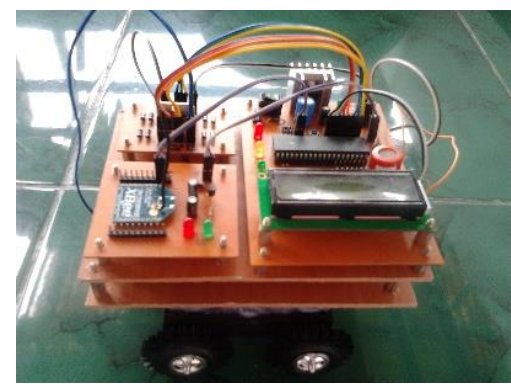

Fig. 13. Mobile robot.

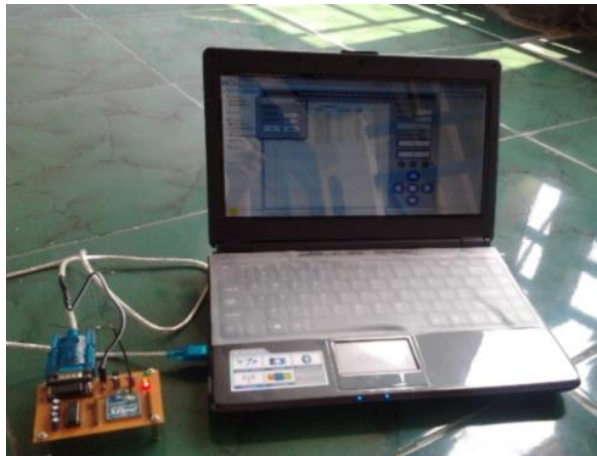

Fig. 14. Ground station.

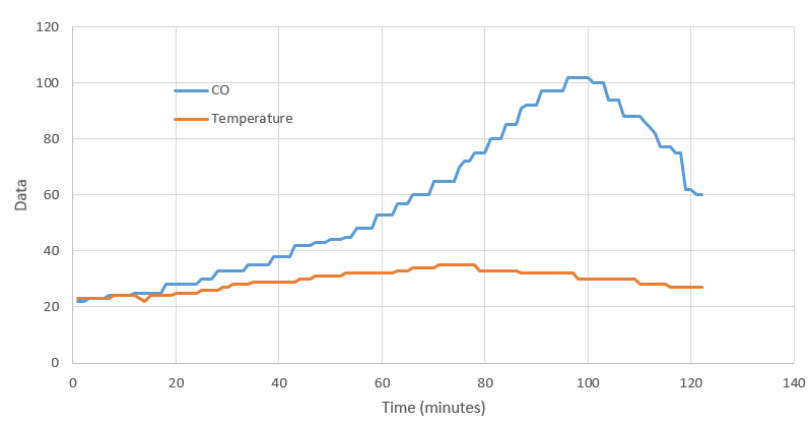

Fig. 15. CO monitoring chart.

We compare the changes in $\mathrm{CO}$ concentration and the temperature values under the same measurement. The $\mathrm{CO}$ gas sensor is tested using cigarette smoke and motor vehicle fumes. The temperature sensor is tested by increasing the temperature using fire. If the $\mathrm{CO}$ ppm is less than 25 , it means the air is normal and fresh. If the CO ppm is between 25 to 50 , it is mean the air has been poisoned with a little $\mathrm{CO}$ gas which can cause some health effects. Otherwise, $\mathrm{CO}$ gas in air is already a high level which can cause headaches, dizziness, fatigue, even loss of consciousness.

\section{a. VII. CONCLUSIONS}

This paper proposes the implementation of a mobile robot as a gas leak detector for early warning on safety. Gas and temperature sensors are used for continuous measurement and monitoring. The measurements were carried out by controlling the movement of a mobile robot to a certain place to take measurements. Besides, all sensor values are used as indicators of changes in gas concentration by applying a threshold level. Data recorded in real-time can be used for further analysis of the sensors detector.

\section{REFERENCES}

[1] A. Milella, G. Cicirelli, and A. Distante, "RFID-assisted mobile robot system for mapping and surveillance of indoor environments," Ind. Rob., vol. 35, no. 2, pp. 143-152, 2008.

[2] G. Cicirelli, A. Milella, and D. Di Paola, "RFID tag localization by using adaptive neurofuzzy inference for mobile robot applications," Ind. Rob., vol. 39, no. 4, pp. 340-348, 2012.

[3] W. Rahmaniar, W. Wang, and H. Chen, "Real-time detection and recognition of multiple moving objects for aerial surveillance," Electronics, vol. 8, no. 12, p. 1373, 2019. 
[4] W. Rahmaniar and W.-J. Wang, "A novel object detection method based on Fuzzy sets theory and SURF," in Proc. of International Conference on System Science and Engineering, 2015, pp. 570-584.

[5] C. Astua, R. Barber, J. Crespo, and A. Jardon, "Object detection techniques applied on mobile robot semantic navigation," Sensors (Switzerland), vol. 14, no. 4, pp. 6734-6757, 2014.

[6] S. Minaeian, J. Liu, and Y. J. Son, "Vision-based target detection and localization via a team of cooperative UAV and UGVs," IEEE Trans. Syst. Man, Cybern. Syst., vol. 46, no. 7, pp. 1005-1016, 2016.

[7] R. C. Luo and C. C. Lai, "Multisensor fusion-based concurrent environment mapping and moving object detection for intelligent service robotics," IEEE Trans. Ind. Electron., vol. 61, no. 8, pp. 4043-4051, 2014.

[8] M. Jin, S. Liu, S. Schiavon, and C. Spanos, "Automated mobile sensing: Towards high-granularity agile indoor environmental quality monitoring," Build. Environ., vol. 127, pp. 268-276, Jan. 2018.

[9] D. Martinez et al., "Ambient intelligence application based on environmental measurements performed with an assistant mobile robot," Sensors, vol. 14, no. 4, pp. 6045-6055, Mar. 2014.

[10]M. Reggente et al., "The DustBot system: Using mobile robots to monitor pollution in pedestrian area," Chem. Eng. Trans., vol. 23, pp. 273-278, 2010.

[11]S. Soldan, G. Bonow, and A. Kroll, "RoboGas Inspector - A mobile robotic system for remote leak sensing and localization in large industrial environments: Overview and first results," IFAC Proc. Vol., vol. 45, no. 8, pp. 33-38, 2012.

[12]V. H. Bennetts, A. J. Lilienthal, P. P. Neumann, and M. Trincavelli, "Mobile robots for localizing gas emission sources on landfill sites: Is bio-inspiration the way to go?," Front. Neuroeng., vol. 4, no. 20, pp. $1-$ 12, 2012.

[13]R. Polvara, M. Trabattoni, T. P. Kucner, E. Schaffernicht, F. Amigoni, and A. J. Lilienthal, "A Next-Best-Smell Approach for Remote Gas Detection with a Mobile Robot," arXiv:1801.06819v1, 2018.

[14]G. Kowadlo and R. A. Russell, "Robot Odor Localization: A Taxonomy and Survey,” Int. J. Rob. Res., vol. 27, no. 8, pp. 869-894, Aug. 2008.

[15]J. Monroy, J.-R. Ruiz-Sarmiento, F.-A. Moreno, F. MelendezFernandez, C. Galindo, and J. Gonzalez-Jimenez, "A semantic-based gas source localization with a mobile robot combining vision and chemical sensing," Sensors, vol. 18, no. 12, p. 4174, Nov. 2018.

[16]A. Loutfi, S. Coradeschi, A. J. Lilienthal, and J. Gonzalez, "Gas distribution mapping of multiple odour sources using a mobile robot," Robotica, vol. 27, no. 2, pp. 311-319, Mar. 2009.

[17]R. Aquino-Santos, D. Martinez-Castro, A. Edwards-Block, and A. F.
Murillo-Piedrahita, "Wireless sensor networks for ambient assisted living," Sensors, vol. 13, no. 12, pp. 16384-16405, Nov. 2013.

[18]Y. F. Chung and C. H. Liu, "Design of a wireless sensor network platform for tele-homecare," Sensors, vol. 13, no. 12, pp. 17156-17175, Dec. 2013.

[19]U. Awasthi and N. R. Chauhan, "Autonomous mobile robot for gas leakage detection and source localization - a review," J. Mater. Sci. Mech. Eng., vol. 3, no. 4, pp. 262-267, 2016.

[20]J. Palacín et al., "Application of an array of metal-oxide semiconductor gas sensors in an assistant personal robot for early gas leak detection," Sensors, vol. 19, no. 9, pp. 1-16, 2019.

[21]D. Martínez et al., "Measuring gas concentration and wind intensity in a turbulent wind tunnel with a mobile robot," J. Sensors, vol. 2016, 2016.

[22]M. Rossi and D. Brunelli, "Autonomous gas detection and mapping with unmanned aerial vehicles," IEEE Trans. Instrum. Meas., vol. 65, no. 4, pp. 18.1-18.8, 2016.

[23]Z. Wang et al., "A sensitive and reliable carbon monoxide monitor for safety-focused applications in coal mine using a 2.33- $\mu \mathrm{m}$ laser diode," IEEE Sens. J., vol. 20, no. 1, pp. 171-177, 2020.

[24]R. Kumar, M. Jaiswal, O. Singh, A. Gupta, M. S. Ansari, and J. Mittal, "Selective and reversible sensing of low concentration of carbon monoxide gas using nb-doped oms-2 nanofibers at room temperature," IEEE Sens. J., vol. 19, no. 17, pp. 7201-7206, 2019.

[25]A. A. S. Ali et al., "Embedded platform for gas applications using hardware/software co-design and RFID," IEEE Sens. J., vol. 18, no. 11, pp. 4633-4642, 2018.

[26]A. Debataraja, A. R. Muchtar, N. L. W. Septiani, B. Yuliarto, Nugrahaauth, and B. Sunendar, "High performance carbon monoxide sensor based on nano composite of SnO2-graphene," IEEE Sens. J., vol. 17, no. 24, pp. 8297-8305, 2017.

[27]Detect Carbon Monoxide, https://www.detectcarbonmonoxide.com/cohealth-risks/

[28]MQ-9 semiconductor sensor for combustible gas, http://hwsensor.com/

[29] LM35 Precision centigrade temperature sensors, http://ti.com/

[30] J. Foster, Xbee CookBook. 2011.

[31]Datasheet ATmega32, http://atmega.com/

[32]L298 dual full-bridge driver, http://sparkfun.com/

[33]F. J. Ferrero Martín, J. C. Campo Rodríguez, J. C. Álvarez Antón, J. C. Viera Pérez, C. B. Viejo, and M. G. Vega, "An electronic instrumentation design project for computer engineering students," IEEE Trans. Educ., vol. 48, no. 3, pp. 472-481, 2005. 